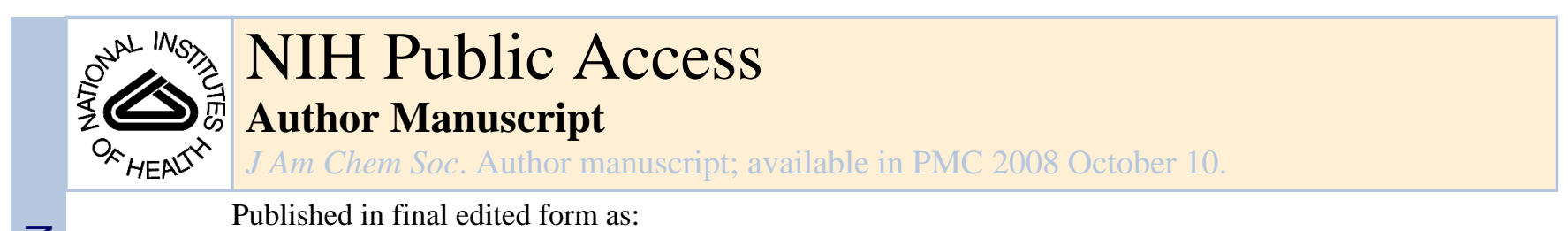

J Am Chem Soc. 2006 March 22; 128(11): 3496-3497. doi:10.1021/ja0582051.

\title{
An a-Helical Peptidomimetic Inhibitor of the HIV-1 Rev-RRE Interaction
}

\author{
Nicholas L. Mills, Matthew D. Daugherty, Alan D. Frankel, and R. Kiplin Guy ${ }^{1, *}$ \\ Departments of Biochemistry and Biophysics, Pharmaceutical Chemistry, and Cellular and \\ Molecular Pharmacology, University of California, San Francisco CA 94143-2280, USA
}

\section{Abstract}

The interaction between the HIV-1 Rev protein and the Rev-Responsive Element (RRE) RNA is an attractive target for anti-viral therapy. We have designed $\alpha$-helical peptidomimetics of Rev-like peptides using sidechain-sidechain macrolactam formation between positions $i$ and $i+4$. One peptidomimetic having an appropriate location, orientation, and length of the macrolactam exhibited both significant helical character and specific RRE binding. This molecule displays two-fold greater RNA specificity than the wild-type Rev peptide and more than 20-fold greater specificity than an uncyclized control peptide. Thus, specific, high affinity recognition of the RRE is feasible utilizing a small, relatively rigid peptidomimetic scaffold.

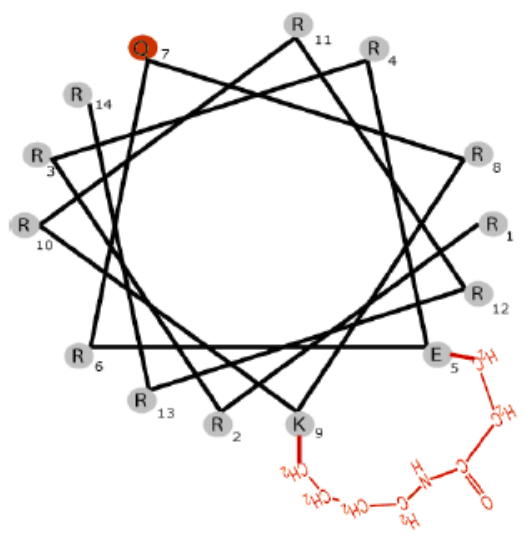

\begin{abstract}
While RNA molecules provide potentially useful targets for drug design, there are few examples of small, relatively rigid molecules able to discriminate between RNA sites with high specificity. One such target is the HIV-1 Rev-Responsive Element (RRE) RNA, which is recognized by the Arginine-Rich Motif (ARM) of the Rev protein ${ }^{2,3}$, primarily via insertion of the $\alpha$-helical ARM into the widened major groove of an asymmetric internal bulge in stemloop IIB. ${ }^{4}$ Binding specificity for RRE correlates with peptide helicity ${ }^{5}$, suggesting that correctly positioning a set of interacting side chains within a rigid structural framework can lead to the desired discrimination. Given that the Rev-RRE interaction facilitates the nuclear export of incompletely processed viral transcripts and is essential for viral replication ${ }^{6}$, it provides an attractive target for anti-HIV therapy.
\end{abstract}

Email: Kip.Guy@StJude.org.

${ }_{1}^{1}$ Present Address: Department of Chemical Biology and Therapeutics, St. Jude Children's Research Hospital, 332 North Lauderdale, Memphis, TN 38105. 
Previous work has shown that pre-stabilizing the Rev ARM $\alpha$-helix within a zinc finger scaffold generates tight RRE-binders. ${ }^{7}$ We wished to create a new class of conformationally constrained $\alpha$-helical peptidomimetics that are short, relatively rigid, and that might be effective competitors of the Rev-RRE interaction. Previously, peptidomimetics of a BIV Tat peptide, which binds to its cognate TAR RNA in a $\beta$-turn conformation, ${ }^{8}$ were designed using headto-tail cyclization to enforce the conformational constraint. ${ }^{9,10}$ Here we report the first $\alpha$ helical peptidomimetics targeted to a viral RNA using macrolactam constraints between amino acid side chains $(i, i+4)$, which have previously been shown to induce helicity in small peptides. 11

We generated peptidomimetics based upon a highly specific RRE binding peptide, $\mathrm{R}_{6} \mathrm{QR}_{7}$, identified in a genetic selection experiment. ${ }^{12}$ When helically stabilized, this peptide binds the RRE with an affinity similar to the Rev ARM and contains a glutamine in place of the asparagine at the central position in the Rev ARM (Fig. 1). The $\mathrm{R}_{6} \mathrm{QR}_{7} \alpha$-helix is presumably oriented further from the RNA major groove than the Rev ARM, potentially allowing more space for a macrolactam constraint.

A series of macrolactam-constrained $\mathrm{R}_{6} \mathrm{QR}_{7}$ peptidomimetics (Fig. 1 and Table 1) was synthesized to investigate both the helical propensity and the specific binding of molecules with different length, location and orientation of the lactam ring. All peptides were synthesized using Fmoc solid phase synthesis with orthogonal $\mathrm{Pd}(0)$ labile protecting groups on the relevant lactam precursors, followed by on-resin lactam formation. ${ }^{13}$ Only one peptidomimetic (8) recognized the RRE with high affinity and specificity. Interestingly, peptide $\mathbf{8}$ exhibits a twofold higher affinity for RRE than a helical Rev peptide stabilized by terminal modifications (17). ${ }^{4}$ Importantly, the unconstrained analog of $\mathbf{8}$, peptide $\mathbf{1 4}$, does not specifically recognize the RRE and thus adding the conformational constraint results in a $>25$-fold increase in binding specificity. Peptide 6, which, like peptide 8, contains a lysine-glutamate macrolactam straddling the essential glutamine, also specifically recognizes the RRE, albeit not as tightly, due to lack of helicity as described below. A helical (Fig. S3) asparagine variant (15) constrained with the same linkage as $\mathbf{8}$, does not specifically bind the RRE. This is consistent with previous results showing the importance of the extra methylene group in the unconstrained $\mathrm{R}_{6} \mathrm{QR}_{7}$ peptide context. ${ }^{11}$

Circular dichroism (CD) spectra of only three peptidomimetics (peptides 4, 8 and 12) exhibited a classical $\alpha$-helical curve shape with dual minima at $222 \mathrm{~nm}$ and $208 \mathrm{~nm}$, whereas the unconstrained $\mathrm{R}_{6} \mathrm{QR}_{7}$ peptide 13 , along with remaining peptidomimetics, exhibited a single minimum at $202 \mathrm{~nm}$, typical of short, unstructured peptides (Fig. 2a and Fig. S3). ${ }^{14}$

Only these three peptidomimetics, having a glutamate-lysine linkage, with glutamate on the $\mathrm{N}$-terminal side, showed any evidence of helicity, with peptide $\mathbf{8}$ exhibiting $33 \%$ helicity. Although all three peptidomimetics are helical by CD, only peptide $\mathbf{8}$ is able to specifically recognize the RRE, illustrating that only the linkage positioned exactly on the opposite side of the essential glutamine is viable for binding. The difference in binding between peptides $\mathbf{8}$ and $\mathbf{6}$ is most likely due to the lack of helical induction in peptide $\mathbf{6}$, based only on the orientation of the macrolactam.

A comparison of the ${ }^{13} \mathrm{C}$ HSQC NMR spectra of the constrained peptide $\mathbf{8}$ and the unconstrained peptide $\mathbf{1 4}$ showed differences in dispersion in the $\mathrm{C} \alpha$-H $\alpha$ region (Fig. 2b), indicating a difference in backbone conformation. Peptide $\mathbf{8}$ revealed numerous peaks with characteristic downfield ${ }^{13} \mathrm{C}$ shifts and upfield ${ }^{1} \mathrm{H}$ shifts consistent with an $\alpha$-helical secondary structure. ${ }^{15}$ Homonuclear 2D TOCSY experiments (see supplement) provided unique assignments for the lysine, glutamate, and glutamine side chains, which all appeared helical based on the dispersion. At least three arginines also showed helical downshifts but could not 
be assigned definitively. The NMR data on peptidomimetic $\mathbf{8}$ are consistent with the $\sim 30 \%$ helicity observed by $\mathrm{CD}$, and further indicates that the helicity is most likely localized within the constrained region of the peptide.

A fluorescence polarization competition assay was used to test whether the conformationally constrained, tight RRE binding peptide $\mathbf{8}$ could efficiently compete for RNA binding with the wild-type Rev peptide. ${ }^{16}$ Peptide $\mathbf{1 7}$, which is $\sim 30 \%$ helical, and has a high affinity for the RRE, was labeled with fluorescein at its C-terminus and titrated with unlabeled competitors (Fig S4).

Of the three helical peptidomimetics $(\mathbf{4}, \mathbf{8}$ and $\mathbf{1 2})$, peptide $\mathbf{8}$ was the most effective competitor, with an $\mathrm{IC}_{50}(\sim 150 \mathrm{nM})$ comparable to its $\mathrm{K}_{\mathrm{d}}(\sim 40 \mathrm{nM})$ (Fig. 3 and Table 1; note that the competition assay was conducted at an RNA concentration four-fold above $\mathrm{K}_{\mathrm{d}}$ ). The other peptidomimetics showed minimal competition, also consistent with the direct binding assays (Fig. 3 and Table 1).

The results presented here indicate that a conformationally-constrained $\alpha$-helical peptidomimetic (peptide 8) can bind the RRE RNA with high affinity and specificity when the constraint is properly located and oriented. The positioning of the constraint on the $\alpha$-helical face opposite the RNA major groove proves essential, as about two-thirds of the $\alpha$-helix is surrounded by RNA in the Rev ARM-RRE complex. ${ }^{3}$ The observation that our designed, relatively short, constrained peptidomimetic is able to recognize the RRE IIB and compete for Rev binding suggests that the approach may be useful for generating other molecules that specifically target the Rev-RRE interaction for anti-viral therapy.

\section{Supplementary Material}

Refer to Web version on PubMed Central for supplementary material.

\section{Acknowledgements}

This work was funded by the NIH (GM 56531), the Sandler Basic Research Foundation, and a Howard Hughes Predoctoral Fellowship to M.D.D.

\section{References}

2. Zapp ML, Green MR. Nature 1989;342:714-6. [PubMed: 2556643]

3. Daly TJ, Cook KS, Gray GS, Maione TE, Rusche JR. Nature 1989;342:816-9. [PubMed: 2481237]

4. Battiste JL, Mao H, Rao NS, Tan R, Muhandiram DR, Kay LE, Frankel AD, Williamson JR. Science 1996;273:1547-51. [PubMed: 8703216]

5. Tan R, Chen L, Buettner JA, Hudson D, Frankel AD. Cell 1993;73:1031-40. [PubMed: 7684657]

6. Malim MH, Hauber J, Le SY, Maizel JV, Cullen BR. Nature 1989;338:254-7. [PubMed: 2784194]

7. McColl DJ, Honchell CD, Frankel AD. Proc Natl Acad Sci U S A 1999;96:9521-6. [PubMed: 10449725]

8. Tamilarasu N, Huq I, Rana TM. Bioorg Med Chem Lett 2000;10:971-4. [PubMed: 10853671]

9. Friedler A, Friedler D, Luedtke NW, Tor Y, Loyter A, Gilon C. J Biol Chem 2000;275:23783-9. [PubMed: 10764789]

10. Runyon ST, Puglisi JD. J Am Chem Soc 2003;125:15704-5. [PubMed: 14677935]

11. Geistlinger TR, Guy RK. J Am Chem Soc 2001;123:1525-6. [PubMed: 11456738]

12. Tan R, Frankel AD. Proc Natl Acad Sci U S A 1998;95:4247-52. [PubMed: 9539722]

13. Geistlinger TR, Guy RK. Methods Enzymol 2003;364:223-46. [PubMed: 14631848]

14. Greenfield NJ. Anal Biochem 1996;235:1-10. [PubMed: 8850540]

15. Wishart DS, Sykes BD. Methods Enzymol 1994;239:363-92. [PubMed: 7830591] 
16. Luedtke NW, Tor Y. Biopolymers 2003;70:103-19. [PubMed: 12925996] 


\section{a) Rev17 Suc- TRQARRNRRRRWRERQR AAAAR-am $\mathbf{R}_{6} \mathbf{Q R}_{7}$ Suc-AAAA RRRRRRQRRRRRRR AAAAR-am}

b)

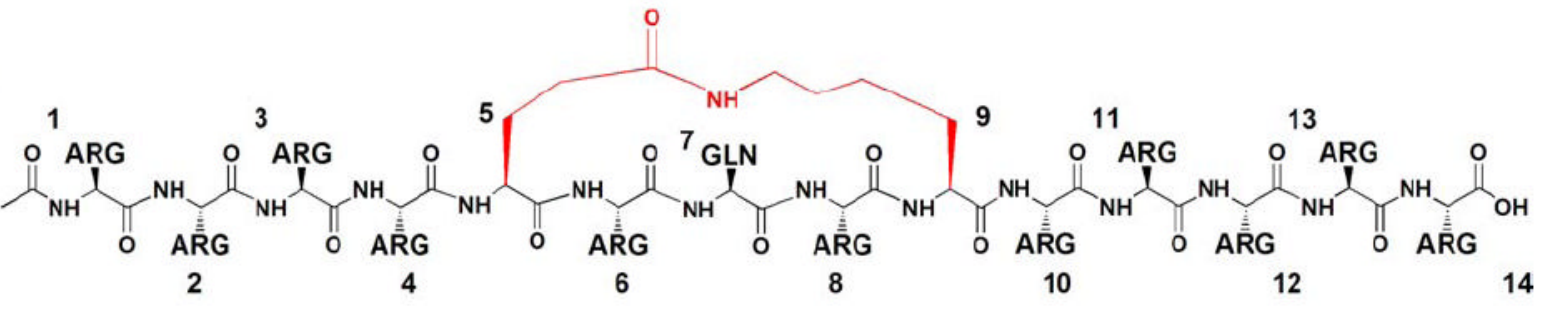

Figure 1.

(a) Sequence comparison of Rev17 and $\mathrm{R}_{6} \mathrm{QR}_{7}$ peptides, helically stabilized by terminal modifications as previously reported. ${ }^{4,11}$ (b) Schematic of $\mathrm{R}_{6} \mathrm{QR}_{7}$ peptidomimetic 8 with $(i$, $i+4)$ macrolactam constraint in red. 
a)

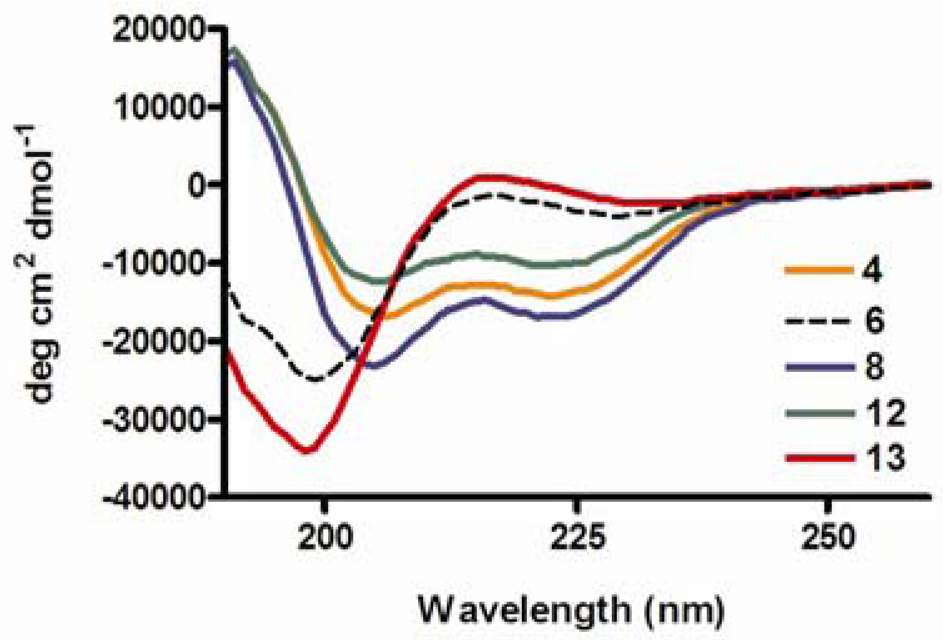

b)

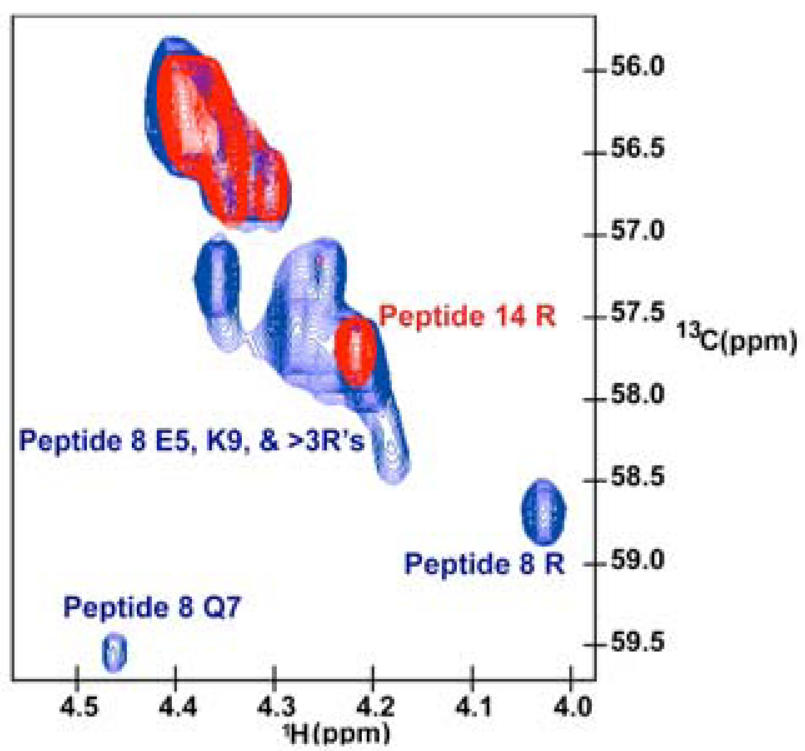

Figure 2.

Structure of $\mathrm{R}_{6} \mathrm{QR}_{7}$ Peptidomimetics. (a) $\mathrm{CD}$ spectra of selected peptidomimetics and unconstrained control peptide (13). CD spectra were recorded at $4^{\circ} \mathrm{C}$ in $10 \mathrm{mM}$ sodium phosphate, $100 \mathrm{mM} \mathrm{KF}, \mathrm{pH} 7.5$. (b) Natural abundance ${ }^{13} \mathrm{C}$ HSQC comparing chemical shifts in the $\mathrm{C} \alpha-\mathrm{H} \alpha$ region between peptide 8 (blue) and uncyclized peptide 14 (red). 


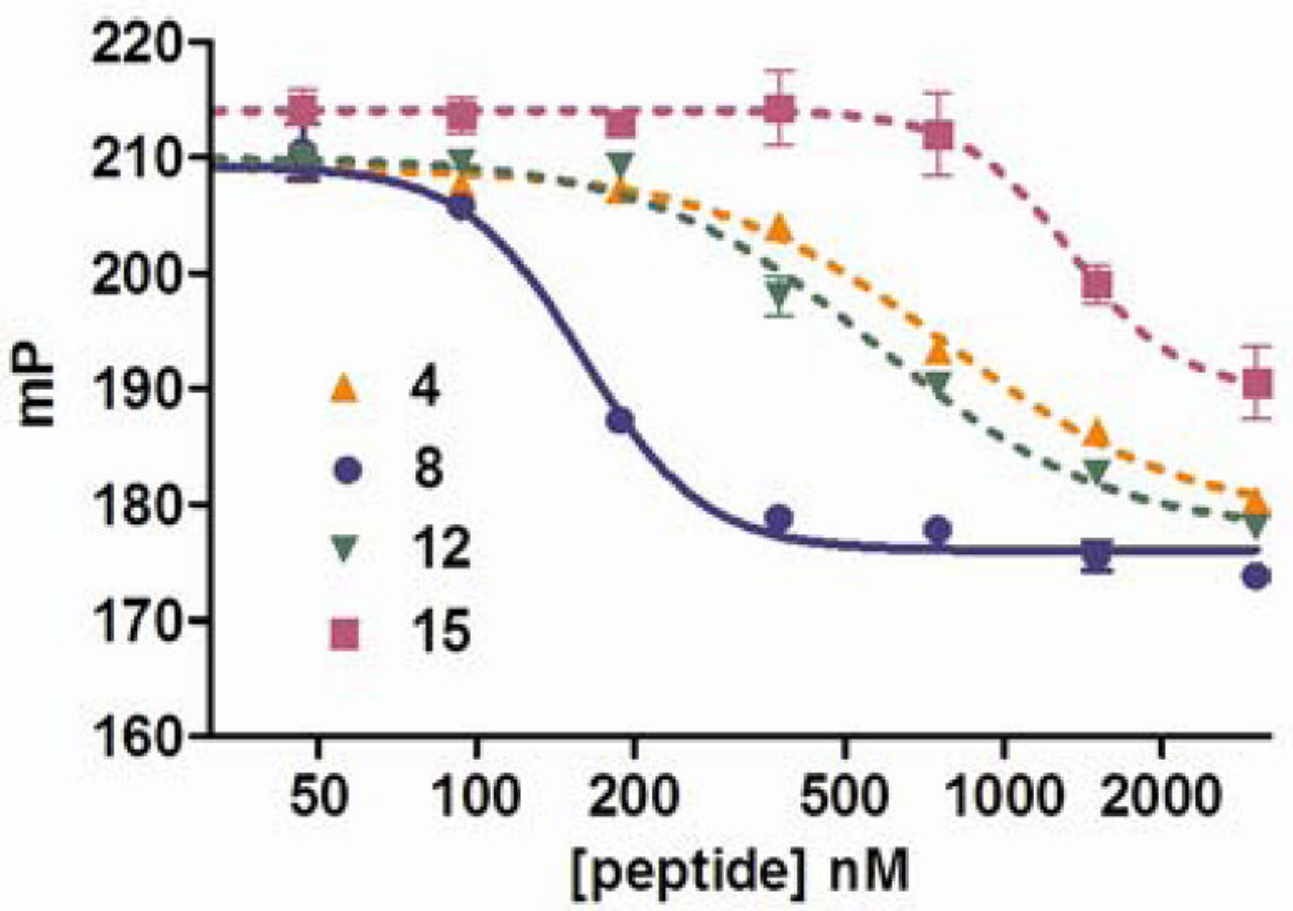

Figure 3.

Fluorescence polarization competition assays with helical peptidomimetics. Assays were performed by titrating competitors into a mixture of RRE RNA and labeled Rev peptide in $30 \mathrm{mM}$ HEPES $\mathrm{pH} 7.5,100 \mathrm{mM} \mathrm{KCl}, 40 \mathrm{mM} \mathrm{NaCl}, 10 \mathrm{mM} \mathrm{NH}_{4} \mathrm{OAc}, 10 \mathrm{mM}$ Guanidinium, $2 \mathrm{mM} \mathrm{MgCl}_{2}, 0.5 \mathrm{mM}$ EDTA, $0.01 \%$ NP-40. ${ }^{16}$ 
Table 1

Composition and Binding of $\mathrm{R}_{6} \mathrm{QR}_{7}$ Peptidomimetics

\begin{tabular}{|c|c|c|c|c|}
\hline \# & Compound & $\mathrm{K}_{\mathrm{sp}}$ & $\mathrm{K}_{\text {nonsp }}$ & Specificity \\
\hline $\mathbf{1}$ & Ac-RRRKRRQDRRRRRR-OH & 800 & 1200 & 1.5 \\
\hline 2 & Ac-RRRKRRQERRRRRR-OH & 1200 & 1600 & 1.3 \\
\hline 3 & Ac-RRRDRRQKRRRRRR-OH & 1200 & 1600 & 1.3 \\
\hline 4 & Ac-RRRERRQKRRRRRR-OH & 700 & 1200 & 1.7 \\
\hline $\mathbf{5}$ & Ac-RRRRKRQRDRRRRR-OH & 1200 & 1600 & 1.3 \\
\hline 6 & Ac-RRRRKRQRERRRRR-OH & 200 & 1200 & 6 \\
\hline 7 & Ac-RRRRDRQRKRRRRR-OH & 700 & 1200 & 1.7 \\
\hline 8 & Ac-RRRRERQRKRRRRR-OH & 45 & 1200 & 26 \\
\hline 9 & $\begin{array}{l}\text { Ac-RRRRRKQRRDRRRR-OH } \\
\end{array}$ & 1200 & 1600 & 1.3 \\
\hline 10 & Ac-RRRRRKQRRERRRR-OH & 1200 & 1200 & 1 \\
\hline 11 & Ac-RRRRRDQRRKRRRR-OH & 1200 & 1200 & 1 \\
\hline 12 & Ac-RRRRREQRRKRRRR-OH & 700 & 1200 & 1.7 \\
\hline 13 & Ac-RRRRRRQRRRRRRR-OH & 1200 & 1200 & 1 \\
\hline 14 & Ac-RRRRERQRKRRRRR-OH & 1600 & 1600 & 1 \\
\hline 15 & Ac-RRRRERNRKRRRRR-OH & 800 & 1200 & 1 \\
\hline 16 & $\mathrm{R}_{6} \mathrm{QR}_{7}$ & 150 & 1200 & 8 \\
\hline 17 & Rev17 & 100 & 1600 & 16 \\
\hline
\end{tabular}

Compounds 1-12 are the designed peptidomimetics; red residues are involved in the macrolactam constraint. Peptide $\mathbf{1 3}$ is a general linear R6QR7 control peptide. RRE binding $\mathrm{K}_{\mathrm{d}}$ 's were determined by electrophoretic mobility shift assays, with specificity defined as the ratio of dissociation constants of wildtype RRE IIB and a C46-G74 mutant RRE, shown not to specifically recognize Rev or R6QR7 peptides. ${ }^{4,11}$ 